\title{
Evaluation of Bovine Digesta as Organic Fertilizer in Maize Using Pot Experiments
}

\author{
S. Meignanalakshmi ${ }^{1}$, M. Charulatha ${ }^{1}$, C. Vennila $^{2}$, P. Tensingh Gnanaraj ${ }^{3}$, \\ A. Serma Saravana Pandian ${ }^{4}$ and K. Vijayarani ${ }^{1}$ \\ ${ }^{1}$ Department of Animal Biotechnology, ${ }^{2}$ Department of Agronomy, Madras Veterinary \\ College, TANUVAS, Chennai-7, India \\ ${ }^{3}$ Instructional Livestock Farm Complex, Madhavaram Milk Colony, \\ TANUVAS, Chennai-51, India \\ ${ }^{4}$ Department of Animal Husbandry Economics, Madras Veterinary College, \\ TANUVAS, Chennai-7, India \\ *Corresponding author
}

\section{A B S T R A C T}

\section{Keywords}

Bovine digesta, organic fertilizer, nutrient release, NPK content and Slaughter house waste

Article Info

Accepted: 06 June 2018 Available Online: 10 July 2018

\begin{abstract}
In the present study Bovine digesta, a major slaughter house waste has been recycled as organic fertilizer. Bovine digesta collected was sun dried for 3 days, powdered and was subjected to physicochemical analysis - BOD, COD, $\mathrm{pH}$ and TDS. Bovine digesta has $1.35 \%$ Nitrogen, $0.72 \%$ Phosphorous and $0.515 \%$ Potassium. Hence, it has been evaluated as organic fertilizer in Maize at various percentages like $75 \%, 100 \%, 125 \%$ and $150 \%$ equated with nitrogen content of urea. Urea and farmyard manure has been used as inorganic and organic fertilizer control for Bovine digesta. The plant growth parameters height, number of leaves, leaf breadth, stem breadth and number of cobs have been studied at $45^{\text {th }}$ and $90^{\text {th }}$ day. Also the pattern of nutrient release (Nitrogen, Phosphorous, Potassium and Carbon) from bovine digesta to soil has been studied by analyzing the soil samples at regular intervals $\left(7,14,21,28,45\right.$ and $90^{\text {th }}$ day). The nutrient content (Nitrogen, Phosphorous and Potassium) in tissues - leaves, stem, root and cobs have also been analyzed. In the present study soil NPK release, Productivity (number of Cobs) and NPK content in cobs was comparable with urea and farmyard control when bovine digesta was added as organic fertilizer.
\end{abstract}

\section{Introduction}

Fertilizers enhance the growth of plants by supplying one or more essential nutrients to plants. Fertilizers provide macronutrients Nitrogen, Phosphorous, Potassium, secondary macronutrients - Calcium, Magnesium, Sulphur and micronutrients - Copper, Iron,
Zinc and Manganese. The macronutrients, consumed in large quantities by plants are present in plant tissues from $0.15 \%-6 \%$ on dry matter basis. Nitrogen present in proteins, DNA and other components like chlorophyll are not available to plants as they are not in fixed form. This makes Nitrogen the most important fertilizer. Fertilizers can be 
classified as organic and inorganic fertilizers. Inorganic fertilizers are synthetic and rapid in action. Commonly used inorganic fertilizers are ammonium nitrate, ammonium sulfate, potash, triple superphosphate and magnesium sulfate. Although inorganic fertilizers provide nutrients to plants in ready form they are subjected to leaching. Higher dosage of inorganic fertilizer not only burns the plants but also may build up toxic soil concentrations that may lead to chemical imbalances. Organic fertilizers though slow in action, provides a healthy growing environment. They contain only plant or animal-based materials that are either a byproduct or end product of naturally occurring substances thus can be easily recycled. The most commonly used organic fertilizers are peat, animal wastes (often from slaughter houses), plant wastes from agriculture, and treated sewage sludge and others like blood meal, bone meal, fish meal. The slaughter house wastes are very difficult to be treated by the industries and hence they should be recycled in an economical way to maintain a good hygiene and proper sanitation. Blood meal, bone meal and fish meal are the products of slaughtered animals which are used as organic fertilizer. Farmyard manure a commonly used organic fertilizer contains $0.5 \%$ nitrogen, $0.2 \%$ phosphorous and $0.5 \%$ potassium in its well decomposed stage. Bovine digesta when analysed for its NPK content showed a higher value when compared to farmyard manure so, in this study bovine digesta a major slaughter house waste has been evaluated as organic fertilizer in Maize plant, a method of low cost recycling.

\section{Materials and Methods}

\section{Preparation of Bovine digesta as organic} fertilizer

Bovine digesta was collected from Perambur slaughter house, Chennai. Bovine digesta was sun dried for about 3 days and powdered. The prepared organic fertilizer was analysed for
NPK content (Ji et al., 2017 and Kalbani et al., 2016). Nitrogen was estimated by using the method proposed by Kirk (1950), Phosphorus by Bray and Kurtz (1945) and Potassium content by Martin et al (1994) and Creed et al (1994). The physicochemical properties - BOD (IS 1993), COD (Rice et al., 2012), pH (Rice et al., 2012) and Total dissolved solids (TDS) (Rice et al., 2012) were analysed.

\section{Evaluation of Bovine digesta as organic fertilizer by pot experiment}

\section{Pot experiment for Maize (Zea mays) \\ Soil sample collection}

Soil samples were collected from University Research Farm, Madhavaram, Chennai- 51 and analysed for Nitrogen (Subbiah and Asija, 1956), Phosphorous (Olsen et al., 1954), Potassium (Stanford and English, 1949), pH (Jackson and Dutton, 1973) and organic carbon (Walkley and Black, 1934) before the start of the experiment (Akbari et al., 2011 and Islam et al., 2017). Analysis was carried out at Krishi Vigyan Kendra (KVK), Veterinary College and research institute, Namakkal.

\section{Seeds}

Maize (Zea mays) seeds were purchased from Department of millets, Tamil Nadu agricultural university (TNAU), Coimbatore.

\section{Pots}

Earthen pots of size 12" (inch) with $10 \mathrm{~kg}$ soil capacity were purchased from Vadivel Tandoori and Pot supplier, Valluvar kottam, Chennai.

\section{Pot experiment}

Based on the Nitrogen content of Bovine digesta (Nitrogen -1.35\%) it was taken at 
different percentage like $75 \%, 100 \%, 125 \%$ and $150 \%$ by equating with Nitrogen percentage in urea. $10 \mathrm{~kg}$ of soil was taken as plain control (Adeniyan et al., 2011). 10kg of soil with farm yard manure as organic fertilizer control and with urea as chemical fertilizer control was taken. Bovine digesta at various percentages like $75,100,125$ and 150 were added to the soil and mixed thoroughly. Seeds (4 seeds per pot) were sowed at a depth of $1.5 \mathrm{~cm}$ (Jayasinghe et al., 2016). After three days, the germination of maize will occur completely. After the plant attained two leaves growth stage, the plants were thinned out to two plants per pot. All the studies were done in triplicates.

\section{Calculation for the amount of fertilizer to be added}

Farmyard manure was added at $56.8 \mathrm{~g} / 10 \mathrm{~kg}$ of soil. Urea at $1.34 \mathrm{~g} / 10 \mathrm{~kg}$ of soil was added (based on the $\mathrm{N}$ content in urea i.e., $\mathrm{N}=46 \%$ )

Urea $=(100 \% * \mathrm{~N}$ recommendation for maize *for $10 \mathrm{~kg}$ of soil)/ (\% $\mathrm{N}$ in urea* $\mathrm{Kg}$ of soil in one hectare).

Bovine digesta as organic fertilizer for maize was added at $75 \%, 100 \%, 125 \%$ and $150 \%$ to $10 \mathrm{~kg}$ of soil (percentage indicates nitrogen content equated with urea).

\section{Plant growth studies with Bovine digesta as organic fertilizer}

Soil samples were collected at regular intervals $\left(7^{\text {th }}, 14^{\text {th }}, 21^{\text {st }}, 28^{\text {th }}, 45^{\text {th }}\right.$ and $90^{\text {th }}$ day) at depth of $0-30 \mathrm{~cm}$ (Alessandro Rovero et al., 2015) and NPK analysis was carried out. The trial was done till $90^{\text {th }}$ day (Adeniyan et al., 2011) and at $45^{\text {th }}$ and $90^{\text {th }}$ day plant height, number of leaves, leaf breadth, stem breadth and number of cobs were measured (Roy et al., 2013). Tissues at $45^{\text {th }}$ and $90^{\text {th }}$ days (leaves, stem, root and cobs) were taken and analyzed for NPK content (Melissa Herman, 2011). Nitrogen in tissues was estimated using Kjeldhal method (Kirk, 1950), phosphorous by using photometric method (Bray and Kurtz, 1945) and Potassium by using colorimetric method (Abul Fadl, 1949).

\section{Results and Discussion}

Bovine digesta converted to organic fertilizer is given in following table.

The physicochemical properties of Bovine digesta is given in Table 1 .

\section{NPK content of bovine digesta}

Nitrogen $-1.35 \%$

Phosphorous $-0.72 \%$

Potassium- $0.515 \%$

Initial $\mathrm{pH}$, organic carbon and NPK of soil at the $0^{\text {th }}$ day is given in Table 3

\section{Plant growth studies to evaluate Bovine digesta as organic fertilizer}

Height of the plant, Number of leaves, Leaf breadth, stem breadth and number of cobs on $45^{\text {th }}$ and $90^{\text {th }}$ day are given in Table 3 and statistical analysis used was Randomized block design.

Randomized block design analysis of evaluation of bovine digesta as organic fertilizer in Maize (Table 3)

Effect of bovine digesta when added as organic fertilizer showed a significant change for height of the plant and number of leaves since the $\mathrm{F}$ value for Height of the plant and number of leaves are highly significant between groups (Plain control, Urea control, farm yard manure control, Bovine digesta $75 \% *, 100 \% *, 125 \% *$ and $150 \% *$ )

Effect of bovine digesta when added as 
organic fertilizer showed a significant change for height of the plant, number of leaves and number of cobs since the $F$ value for Height of the plant, number of leaves and number of cobs are highly significant between days $\left(45^{\text {th }}\right.$ and $90^{\text {th }}$ days). There is no significant change in leaf breadth and stem breadth between groups and between days

Height of the plant and number of leaves Bovine digesta 75\%* showed best results when compared with other groups (Plain control, Urea control, farm yard manure control, Bovine digesta 100\%*,125\%* and $150 \% *)$

No. of cobs- Bovine digesta 100\%*,125\%* and $150 \% *$ showed best results when compared with other groups (Plain control, Urea control, farm yard manure control and Bovine digesta $75 \% *$ )

NPK and organic carbon release in soil on 7 , $14,21,28,45$ and $90^{\text {th }}$ days are given in Table 4 and 5 and statistical analysis used was Randomized block design.

Randomized block design analysis of experiment on evaluation of bovine digesta as organic fertilizer in Maize (Table 4 and 5)

Effect of bovine digesta when added as organic fertilizer showed a significant change for height of the plant and number of leaves since the $F$ value for Nitrogen, Phosphorous and Potassium are highly significant between groups (Plain control, Urea control, farm yard manure control, Bovine digesta $75 \% *, 100 \% *, 125 \% *$ and $150 \% *)$ and days $(7,14,21,28,45$ and 90$)$.

There is no significant change in carbon between groups and between days since the $\mathrm{F}$ value for carbon has no significant change between groups (Plain control, Urea control, farm yard manure control, Bovine digesta75\%*, 100\%*, 125\%* and
$150 \% *)$ and days $(7,14,21,28,45$ and 90$)$.

Nitrogen- Bovine digesta 125\%* showed best results $(276.46 \mathrm{~kg} / \mathrm{ha})$ when compared with other Bovine digesta groups (Bovine digests 75\%*, 100\%* and 125\%*) and Urea control showed best results $(284 \mathrm{~kg} / \mathrm{ha})$ when compared with other groups (Plain control, farm yard manure control, Bovine digesta75\%*, $100 \% *, 125 \% *$ and $150 \% *$ )

Phosphorous- Bovine digesta 100\%* showed best results when compared with other groups (Plain control, Urea control, farm yard manure control, Bovine digesta75\%*,125\%* and 150\%*)

Potassium- Bovine digesta 125\%* showed best results when compared with other groups (Plain control, Urea control, farm yard manure control, Bovine digesta75\%*, $100 \% *$ and $150 \% *$ )

NPK content in root, stem, leaves and cobs on $45^{\text {th }}$ and $90^{\text {th }}$ days are given in Table 6 and statistical analysis used was Randomized block design.

Randomized block design analysis of experiment on evaluation of bovine digesta as organic fertilizer in Maize (Table 6)

Effect of bovine digesta when added as organic fertilizer showed a significant change in Nitrogen, phosphorous and potassium content since the $\mathrm{F}$ value for Nitrogen, Phosphorous and Potassium in root, stem, leaves and cobs are highly significant between groups (Plain control, Urea control, farm yard manure control, Bovine digesta75\%*, 100\%*, 125\%* and $150 \% *)$ and days $\left(45^{\text {th }}\right.$ and $90^{\text {th }}$ day).

Nitrogen in cobs - Bovine digesta 75\%* showed best results (2.47\%) when compared with other Bovine digesta groups $(100 \% *, 125 \% *$ and $150 \% *)$ and Urea control showed best results (2.75\%) when compared with other groups (Plain control, 
farm yard manure control, Bovine digesta75\%*, 100\%*,125\%* and 150\%*)

Phosphorous and potassium in cobs - Bovine digesta $100 \%$ * showed best results $(1.09 \%$ and $1.73 \%)$ when compared with other Bovine digesta groups $(75 \% *, 125 \% *$ and
$150 \% *$ ) and farm yard manure control showed best results (1.4\%) when compared with other groups (Plain control, urea control, Bovine digesta75\%*, $100 \% *, 125 \% *$ and $150 \% *)$.

Table.1 Physicochemical properties of Bovine digesta

\begin{tabular}{|c|c|c|}
\hline Parameters & Results & Methods \\
\hline pH & 6.86 & $4500 \mathrm{H}^{+}$B (Rice et al., 2012) \\
\hline Total dissolved solids(mg/l) & 1510 & IS 3025:P.16:1984:R:2012 (Rice et al., 2012) \\
\hline COD $(\mathbf{m g} / \mathbf{l})$ & 29800 & IS 3025:P.58:2006:R:2012 (Rice et al., 2012) \\
\hline BOD at $\mathbf{2 7} \mathbf{7}^{\mathbf{C}}$ Cor 3days $\mathbf{( m g / l )}$ & 11640 & IS 3025:P.44:1993:R:2009 (BIS, 1993) \\
\hline
\end{tabular}

Table.2 Initial analysis of soil

\begin{tabular}{|c|c|c|}
\hline Parameters & Results & Methods \\
\hline pH & 5.87 & Potentiometry (Jackson and Dutton, 1973) \\
\hline Organic carbon $(\%)$ & 0.3 & Rapid titration method (Walkey and Black 1934) \\
\hline Nitrogen (Kg/ha) & 267 & $\begin{array}{l}\text { Alkaline permanganate Oxidation method (Subbiah and } \\
\text { Asija, 1956) }\end{array}$ \\
\hline Phosphorous (Kg/ha) & 43.2 & Extraction with $0.5 \mathrm{M} \mathrm{NaHCO}_{3}($ Olsen et al., 1954) \\
\hline Potassium (Kg/ha) & 175 & $\begin{array}{l}\text { Extraction with Neutral normal } \mathrm{NH}_{4} \mathrm{OAc} \text { and Flame } \\
\text { photometry (Stanford and English, 1949) }\end{array}$ \\
\hline
\end{tabular}

Table.3 Randomized block design analysis for plant growth studies to evaluate Bovine digesta as organic fertilizer in maize on $45^{\text {th }}$ day

\begin{tabular}{|c|c|c|c|c|c|c|c|c|c|c|c|c|c|c|c|c|c|c|}
\hline \multirow[t]{3}{*}{ Groups } & \multicolumn{2}{|c|}{ Plain control } & \multicolumn{2}{|c|}{$\begin{array}{c}\text { Farmyard } \\
\text { manure control }\end{array}$} & \multicolumn{2}{|c|}{ Urea control } & \multicolumn{2}{|c|}{$\begin{array}{c}\text { Bovine digesta } \\
75 \%\end{array}$} & \multicolumn{2}{|c|}{$\begin{array}{c}\text { Bovine digesta } \\
100 \%\end{array}$} & \multicolumn{2}{|c|}{$\begin{array}{c}\text { Bovine digesta } \\
125 \%\end{array}$} & \multicolumn{2}{|c|}{$\begin{array}{c}\text { Bovine digesta } \\
150 \%\end{array}$} & \multirow{3}{*}{$\begin{array}{l}\text { F value } \\
\text { Between } \\
\text { groups }\end{array}$} & \multirow{3}{*}{$\begin{array}{l}\text { F value } \\
\text { between } \\
\text { days }\end{array}$} & \multirow{3}{*}{$\begin{array}{l}\text { Significance } \\
\text { between } \\
\text { groups }\end{array}$} & \multirow[t]{3}{*}{$\begin{array}{l}\text { Significance } \\
\text { between days }\end{array}$} \\
\hline & \multicolumn{14}{|c|}{ Mean \pm SD } & & & & \\
\hline & $\begin{array}{l}45^{\text {th }} \\
\text { day }\end{array}$ & $\begin{array}{l}90^{\text {th }} \\
\text { day }\end{array}$ & $\begin{array}{l}45^{\text {th }} \\
\text { day }\end{array}$ & $\begin{array}{l}90^{\text {th }} \\
\text { day }\end{array}$ & $\begin{array}{l}45^{\text {th }} \\
\text { day }\end{array}$ & $\begin{array}{l}90^{\text {th }} \\
\text { day }\end{array}$ & $\begin{array}{l}45^{\text {th }} \\
\text { day }\end{array}$ & $\begin{array}{l}90^{\text {th }} \\
\text { day }\end{array}$ & $\begin{array}{l}45^{\text {th }} \\
\text { day }\end{array}$ & $\begin{array}{l}90^{\text {th }} \\
\text { day }\end{array}$ & $\begin{array}{l}45^{\text {th }} \\
\text { day }\end{array}$ & $\begin{array}{l}90^{\text {th }} \\
\text { day }\end{array}$ & $\begin{array}{l}45^{\text {th }} \\
\text { day }\end{array}$ & $\begin{array}{l}90^{\text {th }} \\
\text { day }\end{array}$ & & & & \\
\hline $\begin{array}{l}\mathrm{HP} \text { in } \\
\text { inches }\end{array}$ & $\begin{array}{c}34 \\
\pm \\
6.24\end{array}$ & $\begin{array}{c}68 \\
\pm \\
23 \\
\end{array}$ & $\begin{array}{c}37.66 \\
\pm \\
11.23\end{array}$ & $\begin{array}{c}56.66 \\
\pm \\
5.77\end{array}$ & $\begin{array}{c}40.66 \\
\pm \\
9.23\end{array}$ & $\begin{array}{c}76 \\
\pm \\
17.34\end{array}$ & $\begin{array}{l}47 \\
\pm \\
2\end{array}$ & $\begin{array}{c}121 \\
\pm \\
6.55\end{array}$ & $\begin{array}{c}38 \\
\pm \\
5.29\end{array}$ & $\begin{array}{c}67 \\
\pm \\
11.26\end{array}$ & $\begin{array}{c}40.66 \\
\pm \\
6.02\end{array}$ & $\begin{array}{c}84.66 \\
\pm \\
6.5\end{array}$ & $\begin{array}{l}45 \\
\pm \\
1\end{array}$ & $\begin{array}{c}78.66 \\
\pm \\
13.05\end{array}$ & 7.959 & 138.623 & 0.00 & 0.00 \\
\hline $\begin{array}{c}\mathrm{NL} \text { in } \\
\mathrm{cm}\end{array}$ & $\begin{array}{c}5.66 \\
\pm \\
2.08\end{array}$ & $\begin{array}{c}12 \\
\pm \\
0.00\end{array}$ & $\begin{array}{c}5.66 \\
\pm \\
2.08\end{array}$ & $\begin{array}{c}8.33 \\
\pm \\
1.15\end{array}$ & $\begin{array}{c}10.00 \\
\pm \\
1.00\end{array}$ & $\begin{array}{c}12 \\
\pm \\
2.00\end{array}$ & $\begin{array}{c}11.00 \\
\pm \\
1.00\end{array}$ & $\begin{array}{l}13.33 \\
\pm \\
0.57\end{array}$ & $\begin{array}{c}9.66 \\
\pm \\
1.52\end{array}$ & $\begin{array}{c}12 \\
\pm \\
1.00\end{array}$ & $\begin{array}{c}9.00 \\
\pm \\
3.46\end{array}$ & $\begin{array}{c}12 \\
\pm \\
1.00\end{array}$ & $\begin{array}{l}9.66 \\
\pm \\
1.52\end{array}$ & $\begin{array}{l}13.00 \\
\pm \\
1.00\end{array}$ & 7.148 & 40.33 & 0.00 & 0.00 \\
\hline $\begin{array}{l}\mathrm{LB} \text { in } \\
\mathrm{cm}\end{array}$ & $\begin{array}{c}5.00 \\
\pm \\
0.00\end{array}$ & $\begin{array}{c}5.00 \\
\pm \\
0.00\end{array}$ & $\begin{array}{c}5.00 \\
\pm \\
0.00\end{array}$ & $\begin{array}{c}5.00 \\
\pm \\
0.00\end{array}$ & $\begin{array}{c}5.00 \\
\pm \\
0.00\end{array}$ & $\begin{array}{c}5.00 \\
\pm \\
0.00\end{array}$ & $\begin{array}{c}5.00 \\
\pm \\
0.00\end{array}$ & $\begin{array}{c}5.00 \\
\pm \\
0.00\end{array}$ & $\begin{array}{c}5.00 \\
\pm \\
0.00\end{array}$ & $\begin{array}{c}5.00 \\
\pm \\
0.00\end{array}$ & $\begin{array}{c}5.00 \\
\pm \\
0.00\end{array}$ & $\begin{array}{c}5.00 \\
\pm \\
0.00\end{array}$ & $\begin{array}{c}5.00 \\
\pm \\
0.00\end{array}$ & $\begin{array}{c}5.00 \\
\pm \\
0.00\end{array}$ & - & - & - & - \\
\hline $\begin{array}{l}\mathrm{SB} \text { in } \\
\mathrm{cm}\end{array}$ & $\begin{array}{c}3.00 \\
\pm \\
0.00\end{array}$ & $\begin{array}{c}3.00 \\
\pm \\
0.00\end{array}$ & $\begin{array}{c}3.00 \\
\pm \\
0.00\end{array}$ & $\begin{array}{c}3.00 \\
\pm \\
0.00\end{array}$ & $\begin{array}{c}3.00 \\
\pm \\
0.00\end{array}$ & $\begin{array}{c}3.00 \\
\pm \\
0.00\end{array}$ & $\begin{array}{c}3.00 \\
\pm \\
0.00\end{array}$ & $\begin{array}{c}3.00 \\
\pm \\
0.00\end{array}$ & $\begin{array}{c}3.00 \\
\pm \\
0.00\end{array}$ & $\begin{array}{c}3.00 \\
\pm \\
0.00\end{array}$ & $\begin{array}{c}3.00 \\
\pm \\
0.00\end{array}$ & $\begin{array}{c}3.00 \\
\pm \\
0.00\end{array}$ & $\begin{array}{c}3.00 \\
\pm \\
0.00\end{array}$ & $\begin{array}{c}3.00 \\
\pm \\
0.00\end{array}$ & - & - & - & - \\
\hline $\mathrm{NC}$ & $\begin{array}{c}0.00 \\
\pm \\
0.00\end{array}$ & $\begin{array}{c}2.00 \\
\pm \\
0.00\end{array}$ & $\begin{array}{c}0.00 \\
\pm \\
0.00\end{array}$ & $\begin{array}{c}2.33 \\
\pm \\
0.577\end{array}$ & $\begin{array}{c}0.00 \\
\pm \\
0.00\end{array}$ & $\begin{array}{c}2.66 \\
\pm \\
0.57\end{array}$ & $\begin{array}{c}0.00 \\
\pm \\
0.00\end{array}$ & $\begin{array}{c}2.00 \\
\pm \\
1.00\end{array}$ & $\begin{array}{c}0.00 \\
\pm \\
0.00\end{array}$ & $\begin{array}{c}3.00 \\
\pm \\
1.00\end{array}$ & $\begin{array}{c}0.00 \\
\pm \\
0.00\end{array}$ & $\begin{array}{c}3.00 \\
\pm \\
0.00\end{array}$ & $\begin{array}{c}0.00 \\
\pm \\
0.00\end{array}$ & $\begin{array}{c}3.00 \\
\pm \\
1.00\end{array}$ & 1.212 & 265.091 & 0.329 & 0.00 \\
\hline
\end{tabular}

Where HP- height of the plant, NL- number of leaves, LB- leaf breadth, SB - stem breadth and NC- number of cobs. Where* indicates nitrogen equated with urea. 
Table.4 Randomized block design analysis for Nitrogen, Phosphorous and Potassium release studies from soil when bovine digesta was added as organic fertilizer in Maize

\begin{tabular}{|c|c|c|c|c|c|c|c|c|c|c|c|c|c|c|c|c|c|c|}
\hline \multirow[t]{2}{*}{ Groups } & \multicolumn{6}{|c|}{ Nitrogen } & \multicolumn{6}{|c|}{ Phosphorous } & \multicolumn{6}{|c|}{ Potassium } \\
\hline & $7^{\text {th }}$ day & $14^{\text {th }}$ day & $21^{\text {st }}$ day & $28^{\text {th }}$ day & $45^{\text {th }}$ day & $\begin{array}{l}90^{\text {th }} \\
\text { Day }\end{array}$ & $7^{\text {th }}$ day & $14^{\text {th }}$ day & $21^{\text {st }}$ day & $28^{\text {th }}$ day & $45^{\text {th }}$ day & $\begin{array}{l}90^{\text {th }} \\
\text { Day }\end{array}$ & $7^{\text {th }}$ day & $14^{\text {th }}$ day & $21^{\text {st }}$ day & $\begin{array}{l}28^{\text {th }} \\
\text { day }^{2}\end{array}$ & $\begin{array}{l}45^{\text {th }} \\
\text { day }\end{array}$ & $\begin{array}{l}90^{\text {th }} \\
\text { Day }\end{array}$ \\
\hline \multicolumn{19}{|c|}{ Mean \pm SD } \\
\hline Plain control & $\begin{array}{c}243 \\
\pm \\
2.00\end{array}$ & $\begin{array}{c}238.66 \\
\pm \\
1.66\end{array}$ & $\begin{array}{c}234.56 \\
\pm \\
0.89\end{array}$ & $\begin{array}{c}230.32 \\
\pm \\
0.68\end{array}$ & $\begin{array}{c}225 \\
\pm \\
1.67\end{array}$ & $\begin{array}{c}215.66 \\
\pm \\
2.66\end{array}$ & $\begin{array}{c}18.2 \\
\pm \\
0.2\end{array}$ & $\begin{array}{c}17.7 \\
\pm \\
0.38\end{array}$ & $\begin{array}{c}16.33 \\
\pm \\
0.23\end{array}$ & $\begin{array}{c}14.4 \\
\pm \\
0.6\end{array}$ & $\begin{array}{c}13.36 \\
\pm \\
0.03\end{array}$ & $\begin{array}{c}12.96 \\
\pm \\
0.3\end{array}$ & $\begin{array}{c}176.16 \\
\pm \\
1.04\end{array}$ & $\begin{array}{c}167.70 \\
\pm \\
1.33\end{array}$ & $\begin{array}{c}150.77 \\
\pm \\
1.17\end{array}$ & $\begin{array}{c}134.43 \\
\pm \\
0.51\end{array}$ & $\begin{array}{c}121.9 \\
\pm \\
0.87\end{array}$ & $\begin{array}{c}116.4 \\
\pm \\
1.27\end{array}$ \\
\hline $\begin{array}{c}\text { Farm yard manure } \\
\text { control }\end{array}$ & $\begin{array}{c}244.21 \\
\pm \\
1.21\end{array}$ & $\begin{array}{c}245.22 \\
\pm \\
0.78\end{array}$ & $\begin{array}{c}246.44 \\
\pm \\
1.44\end{array}$ & $\begin{array}{c}247.67 \\
\pm \\
1.34\end{array}$ & $\begin{array}{c}248.66 \\
\pm \\
1.55\end{array}$ & $\begin{array}{c}242 \\
\pm \\
0.67\end{array}$ & $\begin{array}{c}18.26 \\
\pm \\
0.26\end{array}$ & $\begin{array}{c}18.6 \\
\pm \\
0.06\end{array}$ & $\begin{array}{c}19.21 \\
\pm \\
0.35\end{array}$ & $\begin{array}{c}19.7 \\
\pm \\
0.14\end{array}$ & $\begin{array}{c}20.3 \\
\pm \\
0.36\end{array}$ & $\begin{array}{c}19.66 \\
\pm \\
0.34\end{array}$ & $\begin{array}{c}191.0 \\
\pm \\
1.00\end{array}$ & $\begin{array}{c}202.4 \\
\pm \\
0.96\end{array}$ & $\begin{array}{c}210.29 \\
\pm \\
0.86\end{array}$ & $\begin{array}{c}218.37 \\
\pm \\
1.25\end{array}$ & $\begin{array}{c}221.44 \\
\pm \\
0.77\end{array}$ & $\begin{array}{c}219.15 \\
\pm \\
1.23\end{array}$ \\
\hline Urea control & $\begin{array}{c}252 \\
\pm \\
0.24\end{array}$ & $\begin{array}{c}259 \\
\pm \\
1.32\end{array}$ & $\begin{array}{c}264.3 \\
\pm \\
0.64\end{array}$ & $\begin{array}{c}271.5 \\
\pm \\
0.5\end{array}$ & $\begin{array}{c}284 \\
\pm \\
1.35\end{array}$ & $\begin{array}{c}276.76 \\
\pm \\
3.14\end{array}$ & $\begin{array}{c}20.3 \\
\pm \\
0.48\end{array}$ & $\begin{array}{c}20.78 \\
\pm \\
0.44\end{array}$ & $\begin{array}{c}21.2 \\
\pm \\
0.2\end{array}$ & $\begin{array}{c}21.45 \\
\pm \\
0.45\end{array}$ & $\begin{array}{c}21.78 \\
\pm \\
0.22\end{array}$ & $\begin{array}{c}21.33 \\
\pm \\
0.01\end{array}$ & $\begin{array}{c}197.8 \\
\pm \\
0.74\end{array}$ & $\begin{array}{c}203.7 \\
\pm \\
1.83\end{array}$ & $\begin{array}{c}212.52 \\
\pm \\
1.34\end{array}$ & $\begin{array}{c}221.41 \\
\pm \\
0.61\end{array}$ & $\begin{array}{c}225.48 \\
\pm \\
2.34\end{array}$ & $\begin{array}{c}220.96 \\
\pm \\
0.61\end{array}$ \\
\hline $\begin{array}{c}\text { Bovine digesta } \\
75 \%\end{array}$ & $\begin{array}{c}247.66 \\
\pm \\
0.66\end{array}$ & $\begin{array}{c}251 \\
\pm \\
0.67\end{array}$ & $\begin{array}{c}255.66 \\
\pm \\
4.66\end{array}$ & $\begin{array}{c}263.66 \\
\pm \\
1.33\end{array}$ & $\begin{array}{c}272.33 \\
\pm \\
1.33\end{array}$ & $\begin{array}{c}262.66 \\
\pm \\
0.34\end{array}$ & $\begin{array}{c}19.13 \\
\pm \\
0.15\end{array}$ & $\begin{array}{c}19.17 \\
\pm \\
0.08\end{array}$ & $\begin{array}{c}20.12 \\
\pm \\
0.22\end{array}$ & $\begin{array}{c}20.64 \\
\pm \\
0.28\end{array}$ & $\begin{array}{c}20.96 \\
\pm \\
0.04\end{array}$ & $\begin{array}{c}20.63 \\
\pm \\
0.3\end{array}$ & $\begin{array}{c}195.3 \\
\pm \\
0.61\end{array}$ & $\begin{array}{c}202.26 \\
\pm \\
1.17\end{array}$ & $\begin{array}{c}209.7 \\
\pm \\
0.69\end{array}$ & $\begin{array}{c}220.93 \\
\pm \\
0.24\end{array}$ & $\begin{array}{c}219.21 \\
\pm \\
1.33\end{array}$ & $\begin{array}{c}212.99 \\
\pm \\
0.87\end{array}$ \\
\hline $\begin{array}{c}\text { Bovine digesta } \\
100 \%\end{array}$ & $\begin{array}{c}256.33 \\
\pm \\
1.33\end{array}$ & $\begin{array}{c}259.66 \\
\pm \\
0.66\end{array}$ & $\begin{array}{c}263.66 \\
\pm \\
2.34\end{array}$ & $\begin{array}{c}268.66 \\
\pm \\
2.33\end{array}$ & $\begin{array}{c}275.33 \\
\pm \\
3.44\end{array}$ & $\begin{array}{c}267.33 \\
\pm \\
0.67\end{array}$ & $\begin{array}{c}19.5 \\
\pm \\
0.2\end{array}$ & $\begin{array}{c}19.96 \\
\pm \\
0.1\end{array}$ & $\begin{array}{c}20.55 \\
\pm \\
0.02\end{array}$ & $\begin{array}{c}20.89 \\
\pm \\
0.33\end{array}$ & $\begin{array}{c}21.87 \\
\pm \\
0.21\end{array}$ & $\begin{array}{c}20.69 \\
\pm \\
0.09\end{array}$ & $\begin{array}{c}197.07 \\
\pm \\
1.00\end{array}$ & $\begin{array}{c}202.24 \\
\pm \\
1.09\end{array}$ & $\begin{array}{c}211.00 \\
\pm \\
1.00\end{array}$ & $\begin{array}{c}223.7 \\
\pm \\
0.78\end{array}$ & $\begin{array}{c}228.9 \\
\pm \\
0.78\end{array}$ & $\begin{array}{c}223.6 \\
\pm \\
1.41\end{array}$ \\
\hline $\begin{array}{c}\text { Bovine digesta } \\
125 \%\end{array}$ & $\begin{array}{c}253.21 \\
\pm \\
2.93\end{array}$ & $\begin{array}{c}260.33 \\
\pm \\
2.67\end{array}$ & $\begin{array}{c}264.38 \\
\pm \\
1.87\end{array}$ & $\begin{array}{c}270.23 \\
\pm \\
2.59\end{array}$ & $\begin{array}{c}276.46 \\
\pm \\
2.91\end{array}$ & $\begin{array}{c}269.66 \\
\pm \\
1.13\end{array}$ & $\begin{array}{c}19.94 \\
\pm \\
0.14\end{array}$ & $\begin{array}{c}20.15 \\
\pm \\
0.03\end{array}$ & $\begin{array}{c}20.66 \\
\pm \\
0.11\end{array}$ & $\begin{array}{c}20.88 \\
\pm \\
0.1\end{array}$ & $\begin{array}{c}21.37 \\
\pm \\
0.46\end{array}$ & $\begin{array}{c}20.84 \\
\pm \\
0.11\end{array}$ & $\begin{array}{c}199.7 \\
\pm \\
2.13\end{array}$ & $\begin{array}{c}20.4 .03 \\
\pm \\
0.83\end{array}$ & $\begin{array}{c}215.25 \\
\pm \\
1.17\end{array}$ & $\begin{array}{c}225.22 \\
\pm \\
1.07\end{array}$ & $\begin{array}{c}231.33 \\
\pm \\
1.19\end{array}$ & $\begin{array}{c}226.9 \\
\pm \\
0.84\end{array}$ \\
\hline $\begin{array}{c}\text { Bovine digesta } \\
150 \%\end{array}$ & $\begin{array}{c}246.28 \\
\pm \\
2.28\end{array}$ & $\begin{array}{c}251.66 \\
\pm \\
1.66\end{array}$ & $\begin{array}{c}257.5 \\
\pm \\
1.18\end{array}$ & $\begin{array}{c}267.66 \\
\pm \\
0.88\end{array}$ & $\begin{array}{c}273.56 \\
\pm \\
0.56\end{array}$ & $\begin{array}{c}266.78 \\
\pm \\
1.22\end{array}$ & $\begin{array}{c}19.33 \\
\pm \\
0.12\end{array}$ & $\begin{array}{c}19.66 \\
\pm \\
0.11\end{array}$ & $\begin{array}{c}20.1 \\
\pm \\
0.1\end{array}$ & $\begin{array}{c}20.78 \\
\pm \\
0.06\end{array}$ & $\begin{array}{c}21.37 \\
\pm \\
0.16\end{array}$ & $\begin{array}{c}20.57 \\
\pm \\
0.1\end{array}$ & $\begin{array}{c}194.33 \\
\pm \\
1.33\end{array}$ & $\begin{array}{c}201.56 \\
\pm \\
0.69\end{array}$ & $\begin{array}{c}210.15 \\
\pm \\
1.03\end{array}$ & $\begin{array}{c}220.04 \\
\pm \\
1.00\end{array}$ & $\begin{array}{c}225.78 \\
\pm \\
1.22\end{array}$ & $\begin{array}{c}221.53 \\
\pm \\
0.77\end{array}$ \\
\hline $\begin{array}{l}\text { F value Between } \\
\text { Groups }\end{array}$ & \multicolumn{6}{|c|}{961.88} & \multicolumn{6}{|c|}{1039.19} & \multicolumn{6}{|c|}{9678.59} \\
\hline $\begin{array}{l}\text { F value Between } \\
\text { Days }\end{array}$ & \multicolumn{6}{|c|}{204.665} & \multicolumn{6}{|c|}{34.06} & \multicolumn{6}{|c|}{794.29} \\
\hline $\begin{array}{l}\text { SigniFicance } \\
\text { Between } \\
\text { Groups }\end{array}$ & \multicolumn{6}{|c|}{0.000} & \multicolumn{6}{|c|}{0.000} & \multicolumn{6}{|c|}{0.000} \\
\hline $\begin{array}{l}\text { SigniFicance } \\
\text { Between } \\
\text { Days }\end{array}$ & \multicolumn{6}{|c|}{0.000} & \multicolumn{6}{|c|}{0.000} & \multicolumn{6}{|c|}{0.000} \\
\hline
\end{tabular}




\section{Int.J.Curr.Microbiol.App.Sci (2018) 7(7): 506-516}

Table.5 Randomized block design analysis for carbon release studies from soil when bovine digesta was added as organic fertilizer in Maize

\begin{tabular}{|c|c|c|c|c|c|c|c|c|c|c|}
\hline \multirow[t]{2}{*}{ Groups } & \multicolumn{6}{|c|}{$\mathrm{C}$} & \multirow{3}{*}{$\begin{array}{c}\mathrm{F} \text { value } \\
\text { between } \\
\text { groups }\end{array}$} & \multirow{3}{*}{$\begin{array}{c}\text { F value between } \\
\text { days }\end{array}$} & \multirow{3}{*}{$\begin{array}{c}\text { Significance between } \\
\text { groups }\end{array}$} & \multirow{3}{*}{$\begin{array}{c}\text { Significance between } \\
\text { days }\end{array}$} \\
\hline & $7^{\text {th }}$ day & $14^{\text {th }}$ day & $21^{\text {st }}$ day & $28^{\text {th }}$ day & $45^{\text {th }}$ day & $90^{\text {th }}$ day & & & & \\
\hline \multicolumn{7}{|c|}{ Mean \pm SD } & & & & \\
\hline Plain control & $\begin{array}{c}0.28 \\
\pm \\
0.01\end{array}$ & $\begin{array}{c}0.29 \\
\pm \\
0.00\end{array}$ & $\begin{array}{c}0.28 \\
\pm \\
0.02\end{array}$ & $\begin{array}{c}0.28 \\
\pm \\
0.01\end{array}$ & $\begin{array}{c}0.28 \\
\pm \\
0.02\end{array}$ & $\begin{array}{c}0.30 \\
\pm \\
0.01\end{array}$ & \multirow{7}{*}{2.162} & \multirow{7}{*}{1.337} & \multirow{7}{*}{0.023} & \multirow{7}{*}{0.02} \\
\hline $\begin{array}{l}\text { Farm yard } \\
\text { manure control }\end{array}$ & $\begin{array}{c}0.29 \\
\pm \\
0.01\end{array}$ & $\begin{array}{c}0.28 \\
\pm \\
0.02\end{array}$ & $\begin{array}{c}0.30 \\
\pm \\
0.01\end{array}$ & $\begin{array}{c}0.29 \\
\pm \\
0.02\end{array}$ & $\begin{array}{c}0.28 \\
\pm \\
0.01\end{array}$ & $\begin{array}{c}0.30 \\
\pm \\
0.01\end{array}$ & & & & \\
\hline Urea control & $\begin{array}{c}0.29 \\
\pm \\
0.02\end{array}$ & $\begin{array}{c}0.31 \\
\pm \\
0.02\end{array}$ & $\begin{array}{c}0.29 \\
\pm \\
0.02\end{array}$ & $\begin{array}{c}0.29 \\
\pm \\
0.01\end{array}$ & $\begin{array}{c}0.29 \\
\pm \\
0.02\end{array}$ & $\begin{array}{c}0.29 \\
\pm \\
0.01\end{array}$ & & & & \\
\hline $\begin{array}{l}\text { Bovine digesta } \\
75 \%\end{array}$ & $\begin{array}{c}0.31 \\
\pm \\
0.01\end{array}$ & $\begin{array}{c}0.28 \\
\pm \\
0.01\end{array}$ & $\begin{array}{c}0.30 \\
\pm \\
0.01\end{array}$ & $\begin{array}{c}0.30 \\
\pm \\
0.01\end{array}$ & $\begin{array}{c}0.29 \\
\pm \\
0.01\end{array}$ & $\begin{array}{c}0.29 \\
\pm \\
0.02\end{array}$ & & & & \\
\hline $\begin{array}{l}\text { Bovine digesta } \\
100 \%\end{array}$ & $\begin{array}{c}0.31 \\
\pm \\
0.02\end{array}$ & $\begin{array}{c}0.28 \\
\pm \\
0.02\end{array}$ & $\begin{array}{c}0.31 \\
\pm \\
0.01\end{array}$ & $\begin{array}{c}0.30 \\
\pm \\
0.02\end{array}$ & $\begin{array}{c}0.29 \\
\pm \\
0.02\end{array}$ & $\begin{array}{c}0.30 \\
\pm \\
0.02\end{array}$ & & & & \\
\hline $\begin{array}{l}\text { Bovine digesta } \\
125 \%\end{array}$ & $\begin{array}{c}0.29 \\
\pm \\
0.01\end{array}$ & $\begin{array}{c}0.30 \\
\pm \\
0.01\end{array}$ & $\begin{array}{c}0.30 \\
\pm \\
0.01\end{array}$ & $\begin{array}{c}0.28 \\
\pm \\
0.01\end{array}$ & $\begin{array}{c}0.29 \\
\pm \\
0.01\end{array}$ & $\begin{array}{c}0.30 \\
\pm \\
0.02\end{array}$ & & & & \\
\hline $\begin{array}{l}\text { Bovine digesta } \\
150 \%\end{array}$ & $\begin{array}{c}0.28 \\
\pm \\
0.01\end{array}$ & $\begin{array}{c}0.28 \\
\pm \\
0.02\end{array}$ & $\begin{array}{c}0.30 \\
\pm \\
0.02\end{array}$ & $\begin{array}{c}0.31 \\
\pm \\
0.01\end{array}$ & $\begin{array}{c}0.30 \\
\pm \\
0.02\end{array}$ & $\begin{array}{c}0.29 \\
\pm \\
0.01\end{array}$ & & & & \\
\hline
\end{tabular}


Table.6 Randomized block design analysis for Nitrogen, Phosphorous and Potassium uptake studies in root, leaves, stem and cobs when bovine digesta was added as organic fertilizer in Maize

\begin{tabular}{|c|c|c|c|c|c|c|c|c|c|c|c|c|c|c|c|c|c|c|}
\hline \multirow[t]{3}{*}{ Groups } & \multicolumn{2}{|c|}{ Plain control } & \multicolumn{2}{|c|}{$\begin{array}{c}\text { Farmyard manure } \\
\text { control }\end{array}$} & \multicolumn{2}{|c|}{ Urea control } & \multicolumn{2}{|c|}{ Bovine digesta $75 \%$} & \multicolumn{2}{|c|}{ Bovine digesta $100 \%$} & \multicolumn{2}{|c|}{ Bovine digesta $125 \%$} & \multicolumn{2}{|c|}{ Bovine digesta $150 \%$} & \multirow{3}{*}{$\begin{array}{l}\text { F value } \\
\text { Between } \\
\text { groups }\end{array}$} & \multirow{3}{*}{$\begin{array}{l}\text { F value } \\
\text { between } \\
\text { days }\end{array}$} & \multirow{3}{*}{$\begin{array}{l}\text { Significance } \\
\text { between } \\
\text { groups }\end{array}$} & \multirow{3}{*}{$\begin{array}{l}\text { Significance } \\
\text { between } \\
\text { days }\end{array}$} \\
\hline & & & & & & & & SD & & & & & & & & & & \\
\hline & $45^{\text {th }}$ day & $90^{\text {th }}$ day & $45^{\text {th }}$ day & $90^{\text {th }}$ day & $45^{\text {th }}$ day & $90^{\text {th }}$ day & $45^{\text {th }}$ day & $90^{\text {th }}$ day & $45^{\text {th }}$ day & $90^{\text {th }}$ day & $45^{\text {th }}$ day & $90^{\text {th }}$ day & $45^{\text {th }}$ day & $90^{\text {th }}$ day & & & & \\
\hline $\mathrm{RN}$ & $\begin{array}{c}0.25 \\
\pm \\
0.01\end{array}$ & $\begin{array}{c}0.28 \\
\pm \\
0.01\end{array}$ & $\begin{array}{c}0.21 \\
\pm \\
0.01\end{array}$ & $\begin{array}{c}0.20 \\
\pm \\
0.01\end{array}$ & $\begin{array}{c}0.16 \\
\pm \\
0.01\end{array}$ & $\begin{array}{c}0.25 \\
\pm \\
0.01\end{array}$ & $\begin{array}{c}0.38 \\
\pm \\
0.01\end{array}$ & $\begin{array}{c}0.24 \\
\pm \\
0.01\end{array}$ & $\begin{array}{c}0.2 \\
\pm \\
0.01\end{array}$ & $\begin{array}{c}0.25 \\
\pm \\
0.01\end{array}$ & $\begin{array}{c}0.27 \\
\pm \\
0.01\end{array}$ & $\begin{array}{c}0.19 \\
\pm \\
0.01\end{array}$ & $\begin{array}{c}0.32 \\
\pm \\
0.02\end{array}$ & $\begin{array}{c}0.27 \\
\pm \\
0.01\end{array}$ & 188.17 & 34.615 & 0.00 & 0.00 \\
\hline $\mathrm{RP}$ & $\begin{array}{c}0.06 \\
\pm \\
0.01\end{array}$ & $\begin{array}{c}0.19 \\
\pm \\
0.01\end{array}$ & $\begin{array}{c}0.14 \\
\pm \\
0.01\end{array}$ & $\begin{array}{c}0.2 \\
\pm \\
0.01\end{array}$ & $\begin{array}{c}0.05 \\
\pm \\
0.01\end{array}$ & $\begin{array}{c}0.2 \\
\pm \\
0.01\end{array}$ & $\begin{array}{c}0.52 \\
\pm \\
0.01\end{array}$ & $\begin{array}{c}0.18 \\
\pm \\
0.01\end{array}$ & $\begin{array}{c}0.25 \\
\pm \\
0.01\end{array}$ & $\begin{array}{c}0.22 \\
\pm \\
0.00\end{array}$ & $\begin{array}{c}0.32 \\
\pm \\
0.01\end{array}$ & $\begin{array}{c}0.13 \\
\pm \\
0.01\end{array}$ & $\begin{array}{c}0.23 \\
\pm \\
0.01\end{array}$ & $\begin{array}{c}0.12 \\
\pm \\
0.01\end{array}$ & 544.90 & 357.14 & 0.00 & 0.00 \\
\hline RK & $\begin{array}{c}0.51 \\
\pm \\
0.02\end{array}$ & $\begin{array}{c}0.66 \\
\pm \\
0.01\end{array}$ & $\begin{array}{c}0.47 \\
\pm \\
0.01\end{array}$ & $\begin{array}{c}0.60 \\
\pm \\
0.01\end{array}$ & $\begin{array}{c}0.51 \\
\pm \\
0.01\end{array}$ & $\begin{array}{c}0.54 \\
\pm \\
0.01\end{array}$ & $\begin{array}{c}0.39 \\
\pm \\
0.01\end{array}$ & $\begin{array}{c}0.60 \\
\pm \\
0.01\end{array}$ & $\begin{array}{c}1.19 \\
\pm \\
0.02\end{array}$ & $\begin{array}{c}0.57 \\
\pm \\
0.02\end{array}$ & $\begin{array}{c}0.81 \\
\pm \\
0.01\end{array}$ & $\begin{array}{c}0.61 \\
\pm \\
0.01\end{array}$ & $\begin{array}{c}0.88 \\
\pm \\
0.02\end{array}$ & $\begin{array}{c}0.61 \\
\pm \\
0.01\end{array}$ & 1043.1 & 589.79 & 0.00 & 0.00 \\
\hline $\mathrm{SN}$ & $\begin{array}{c}0.9 \\
\pm \\
0.01\end{array}$ & $\begin{array}{c}1.96 \\
\pm \\
0.01\end{array}$ & $\begin{array}{c}0.83 \\
\pm \\
0.01\end{array}$ & $\begin{array}{c}1.94 \\
\pm \\
0.01\end{array}$ & $\begin{array}{c}1.02 \\
\pm \\
0.03\end{array}$ & $\begin{array}{c}1.59 \\
\pm \\
0.01\end{array}$ & $\begin{array}{c}1.09 \\
\pm \\
0.01\end{array}$ & $\begin{array}{c}1.51 \\
\pm \\
0.01\end{array}$ & $\begin{array}{c}1.01 \\
\pm \\
0.01\end{array}$ & $\begin{array}{c}1.62 \\
\pm \\
0.01\end{array}$ & $\begin{array}{c}1.06 \\
\pm \\
0.01\end{array}$ & $\begin{array}{c}1.46 \\
\pm \\
0.01\end{array}$ & $\begin{array}{c}1.00 \\
\pm \\
0.01\end{array}$ & $\begin{array}{c}1.38 \\
\pm \\
0.01\end{array}$ & 286.03 & 33125 & 0.00 & 0.00 \\
\hline SP & $\begin{array}{c}0.23 \\
\pm \\
0.01\end{array}$ & $\begin{array}{c}0.59 \\
\pm \\
0.01\end{array}$ & $\begin{array}{c}0.50 \\
\pm \\
0.01\end{array}$ & $\begin{array}{c}1.05 \\
\pm \\
0.01\end{array}$ & $\begin{array}{c}0.21 \\
\pm \\
0.01\end{array}$ & $\begin{array}{c}0.67 \\
\pm \\
0.01\end{array}$ & $\begin{array}{c}0.41 \\
\pm \\
0.01\end{array}$ & $\begin{array}{c}0.66 \\
\pm \\
0.01\end{array}$ & $\begin{array}{c}0.56 \\
\pm \\
0.02\end{array}$ & $\begin{array}{c}0.66 \\
\pm \\
0.01\end{array}$ & $\begin{array}{c}0.65 \\
\pm \\
0.01\end{array}$ & $\begin{array}{c}0.54 \\
\pm \\
0.01\end{array}$ & $\begin{array}{c}0.32 \\
\pm \\
0.02\end{array}$ & $\begin{array}{c}0.47 \\
\pm \\
0.01\end{array}$ & 1248.4 & 7476.0 & 0.00 & 0.00 \\
\hline SK & $\begin{array}{c}1.81 \\
\pm \\
0.01\end{array}$ & $\begin{array}{c}2.83 \\
\pm \\
0.01\end{array}$ & $\begin{array}{c}2.28 \\
\pm \\
0.01\end{array}$ & $\begin{array}{c}4.02 \\
\pm \\
0.02\end{array}$ & $\begin{array}{c}1.76 \\
\pm \\
0.01\end{array}$ & $\begin{array}{c}4.68 \\
\pm \\
0.01\end{array}$ & $\begin{array}{c}1.36 \\
\pm \\
0.03\end{array}$ & $\begin{array}{c}2.26 \\
\pm \\
0.01\end{array}$ & $\begin{array}{c}2.67 \\
\pm \\
0.01\end{array}$ & $\begin{array}{c}1.71 \\
\pm \\
0.02\end{array}$ & $\begin{array}{c}3.14 \\
\pm \\
0.01\end{array}$ & $\begin{array}{c}1.39 \\
\pm \\
0.01\end{array}$ & $\begin{array}{c}3.93 \\
\pm \\
0.01\end{array}$ & $\begin{array}{c}1.92 \\
\pm \\
0.01\end{array}$ & 10735.6 & 4512.5 & 0.00 & 0.00 \\
\hline $\mathrm{LN}$ & $\begin{array}{c}1.66 \\
\pm \\
0.01\end{array}$ & $\begin{array}{c}1.5 \\
\pm \\
0.01\end{array}$ & $\begin{array}{c}1.28 \\
\pm \\
0.01\end{array}$ & $\begin{array}{c}1.69 \\
\pm \\
0.01\end{array}$ & $\begin{array}{c}1.3 \\
\pm \\
0.01\end{array}$ & $\begin{array}{c}1.19 \\
\pm \\
0.01\end{array}$ & $\begin{array}{c}1.35 \\
\pm \\
0.02\end{array}$ & $\begin{array}{c}1.21 \\
\pm \\
0.01\end{array}$ & $\begin{array}{c}1.32 \\
\pm \\
0.02\end{array}$ & $\begin{array}{c}1.61 \\
\pm \\
0.01\end{array}$ & $\begin{array}{c}1.76 \\
\pm \\
0.01\end{array}$ & $\begin{array}{c}1.31 \\
\pm \\
0.01\end{array}$ & $\begin{array}{c}1.38 \\
\pm \\
0.01\end{array}$ & $\begin{array}{c}1.46 \\
\pm \\
0.01\end{array}$ & 993.76 & 12.1 & 0.00 & 0.00 \\
\hline LP & $\begin{array}{c}0.22 \\
\pm \\
0.01\end{array}$ & $\begin{array}{c}0.55 \\
\pm \\
0.01\end{array}$ & $\begin{array}{c}0.27 \\
\pm \\
0.01\end{array}$ & $\begin{array}{c}0.93 \\
\pm \\
0.01\end{array}$ & $\begin{array}{c}0.25 \\
\pm \\
0.01\end{array}$ & $\begin{array}{c}0.53 \\
\pm \\
0.01\end{array}$ & $\begin{array}{c}0.31 \\
\pm \\
0.01\end{array}$ & $\begin{array}{c}0.40 \\
\pm \\
0.01\end{array}$ & $\begin{array}{c}0.31 \\
\pm \\
0.01\end{array}$ & $\begin{array}{c}0.57 \\
\pm \\
0.01\end{array}$ & $\begin{array}{c}0.43 \\
\pm \\
0.01\end{array}$ & $\begin{array}{c}0.45 \\
\pm \\
0.01\end{array}$ & $\begin{array}{c}0.26 \\
\pm \\
0.01\end{array}$ & $\begin{array}{c}0.35 \\
\pm \\
0.01\end{array}$ & 817.07 & 9937.9 & 0.00 & 0.00 \\
\hline LK & $\begin{array}{c}1.23 \\
\pm \\
0.01\end{array}$ & $\begin{array}{c}2.89 \\
\pm \\
0.01\end{array}$ & $\begin{array}{c}0.86 \\
\pm \\
0.01\end{array}$ & $\begin{array}{c}3.98 \\
\pm \\
0.01\end{array}$ & $\begin{array}{c}1.17 \\
\pm \\
0.01\end{array}$ & $\begin{array}{c}2.77 \\
\pm \\
0.01\end{array}$ & $\begin{array}{c}1.76 \\
\pm \\
0.02\end{array}$ & $\begin{array}{c}2.95 \\
\pm \\
0.01\end{array}$ & $\begin{array}{c}2.12 \\
\pm \\
0.01\end{array}$ & $\begin{array}{c}1.36 \\
\pm \\
0.01\end{array}$ & $\begin{array}{c}2.56 \\
\pm \\
0.01\end{array}$ & $\begin{array}{c}1.64 \\
\pm \\
0.02\end{array}$ & $\begin{array}{c}2.07 \\
\pm \\
0.02\end{array}$ & $\begin{array}{c}1.22 \\
\pm \\
0.01\end{array}$ & 3574.5 & 39364 & 0.00 & 0.00 \\
\hline $\mathrm{CN}$ & $\begin{array}{c}0.00 \\
\pm \\
0.00\end{array}$ & $\begin{array}{c}2.08 \\
\pm \\
0.01\end{array}$ & $\begin{array}{c}0.00 \\
\pm \\
0.00\end{array}$ & $\begin{array}{c}2.40 \\
\pm \\
0.02\end{array}$ & $\begin{array}{c}0.00 \\
\pm \\
0.00\end{array}$ & $\begin{array}{c}2.75 \\
\pm \\
0.01\end{array}$ & $\begin{array}{c}0.00 \\
\pm \\
0.00\end{array}$ & $\begin{array}{c}2.47 \\
\pm \\
0.01\end{array}$ & $\begin{array}{c}0.00 \\
\pm \\
0.00\end{array}$ & $\begin{array}{c}2.12 \\
\pm \\
0.01\end{array}$ & $\begin{array}{c}0.00 \\
\pm \\
0.00\end{array}$ & $\begin{array}{c}2.24 \\
\pm \\
0.01\end{array}$ & $\begin{array}{c}0.00 \\
\pm \\
0.00\end{array}$ & $\begin{array}{c}2.27 \\
\pm \\
0.288\end{array}$ & 3420.7 & 133840 & 0.00 & 0.00 \\
\hline $\mathrm{CP}$ & $\begin{array}{c}0.00 \\
\pm \\
0.00\end{array}$ & $\begin{array}{c}0.91 \\
\pm \\
0.01\end{array}$ & $\begin{array}{c}0.00 \\
\pm \\
0.00\end{array}$ & $\begin{array}{c}1.40 \\
\pm \\
0.02\end{array}$ & $\begin{array}{c}0.00 \\
\pm \\
0.00\end{array}$ & $\begin{array}{c}1.36 \\
\pm \\
0.01\end{array}$ & $\begin{array}{c}0.00 \\
\pm \\
0.00\end{array}$ & $\begin{array}{c}0.59 \\
\pm \\
0.02\end{array}$ & $\begin{array}{c}0.00 \\
\pm \\
0.00\end{array}$ & $\begin{array}{c}1.09 \\
\pm \\
0.01\end{array}$ & $\begin{array}{c}0.00 \\
\pm \\
0.00\end{array}$ & $\begin{array}{c}0.83 \\
\pm \\
0.01\end{array}$ & $\begin{array}{c}0.00 \\
\pm \\
0.00\end{array}$ & $\begin{array}{c}0.84 \\
\pm \\
0.01\end{array}$ & 2448.0 & 193017 & 0.00 & 0.00 \\
\hline CK & $\begin{array}{c}0.00 \\
\pm \\
0.00\end{array}$ & $\begin{array}{c}1.98 \\
\pm \\
0.01\end{array}$ & $\begin{array}{c}0.00 \\
\pm \\
0.00\end{array}$ & $\begin{array}{c}2.03 \\
\pm \\
0.01\end{array}$ & $\begin{array}{c}0.00 \\
\pm \\
0.00\end{array}$ & $\begin{array}{c}1.34 \\
\pm \\
0.02\end{array}$ & $\begin{array}{c}0.00 \\
\pm \\
0.00\end{array}$ & $\begin{array}{c}1.67 \\
\pm \\
0.01\end{array}$ & $\begin{array}{c}0.00 \\
\pm \\
0.00\end{array}$ & $\begin{array}{c}1.73 \\
\pm \\
0.03\end{array}$ & $\begin{array}{c}0.00 \\
\pm \\
0.00\end{array}$ & $\begin{array}{c}1.34 \\
\pm \\
0.01\end{array}$ & $\begin{array}{c}0.00 \\
\pm \\
0.00\end{array}$ & $\begin{array}{c}1.73 \\
\pm \\
0.287\end{array}$ & 1068.8 & 245226 & 0.00 & 0.00 \\
\hline
\end{tabular}

Where RN- root Nitrogen, RP- Root Phosphorous, RK - Root potassium, SN- stem nitrogen, SP- stem phosphorous, SK- stem potassium, LN- leaf nitrogen, LP-

leaf phosphorous, $\mathrm{LK}$ - leaf potassium, $\mathrm{CN}$ - cob nitrogen, $\mathrm{CP}$ - cob phosphorous and $\mathrm{CK}$ - cob potassium. Where* indicates nitrogen equated with urea. 
Bovine digesta when analysed for NPK content showed a higher values of nitrogen, phosphorous and potassium than farmyard manure. The present study reveals that bovine digesta a major slaughter house waste can be recycled and used as organic fertilizer replacing commonly used fertilizer. Bovine digesta $75 \% *$ showed best results in height of the plant as well as number of leaves when compared with other groups (Plain control, Urea control, farm yard manure control, Bovine digesta 100\%*, 125\%* and 150\%*). Improvement in height and number of leaves was also reported in chilli, tomato and brinjal when another type of organic fertilizer BBRDM (bovine blood rumen digesta mixture) has been used by Roy et al., 2013. Similar findings have been reported by Oyedeji et al., 2014 where poultry manure when used as organic fertilizer improved the plant height as well as the number of leaves in Amaranths deflexus and Amaranths hybridus. Bovine digesta 100\%*, 125\%* and 150\%* showed best results in producing number of cobs when compared with other groups (Plain control, Urea control, farm yard manure control and Bovine digesta 75\%*). Similar findings have been reported by Farhad et al., 2009 and Enujeke, 2013 for poultry manure, when there is an increase in the dosage of poultry manure which was used as an organic fertilizer, the number of cob production have been improved. Similar findings have been reported by Okoroafor et al., 2013 where poultry droppings and pig dung when used as organic fertilizer improved the number of cob production in maize when compared with plain control. Bovine digesta 100\%* and $125 \%$ * showed best results in soil phosphorous and potassium release when compared with other groups (Plain control, Urea control, farm yard manure control, Bovine digesta $75 \% *$ and $150 \% *$ ). Bovine digesta $125 \% *$ showed best results (276.46 $\mathrm{kg} / \mathrm{ha}$ ) in nitrogen release in soil and Bovine digesta $75 \% *$ showed best results in nitrogen content in cobs when compared with other Bovine digesta groups (Bovine digests 75\%*, $100 \% *$ and 125\%*). Urea control showed best results in nitrogen release $(284 \mathrm{~kg} / \mathrm{ha})$ and nitrogen content in cobs $(2.75 \%)$ when compared with other groups (Plain control, farm yard manure control, Bovine digesta $75 \% *, 100 \% *, 125 \% *$ and $150 \% *)$. Bovine digesta $100 \% *$ showed best results in phosphorous content in cobs $(1.09 \%)$ and potassium content in cobs $(1.73 \%)$ when compared with other Bovine digesta groups (Bovine digests 75\%*, 125\%* and 150\%*). Farm yard manure control showed best results in phosphorous content $(1.4 \%)$ in cobs and potassium content $(2.03 \%)$ in cobs when compared with other groups (Plain control, urea control, Bovine digesta75\%*, 100\%*, $125 \% *$ and $150 \% *$ ). Similarly an average NPK content in grains have been found and reported when cattle manure was used as fertilizer when compared to inorganic fertilizer by Melissa Herman (2011).

In the present study it is concluded that the Bovine digesta $125 \% *$ (nitrogen equated with urea) showed best results in terms of soil Nitrogen, phosphorous and potassium release as well as the number of cobs produced.

\section{Acknowledgment}

The authors express their gratitude to The State planning commission - Tamil Nadu state land use research board (SPC-TNSLURB) for financially supporting this research project.

\section{References}

Abul Fadl, M.A.M. 1949. Colorimetric determination of potassium by FolinCiocalteu phenol reagent. Biochem J. 44(3): 282-285.

Adeniyan, O.N., Ojo, A.O., Akinbode, O.A. and Adediran, J.A. 2011. Comparative study of different organic manures and 
NPK fertilizer for improvement of soil chemical properties and dry matter yield of maize in two different soils. Journal of Soil Science and Environmental Management. 2(1): 913.

Akbari, P., Ghalavand, A., Modarres Sanavy, A.M. and Agha Alikhani, M. 2011. The effect of biofertilizers, nitrogen fertilizer and farmyard manure on grain yield and seed quality of sunflower (Helianthus annus L.). Journal of Agricultural Technology. 7(1): 173-184.

Alessandro Rovero, Marco Vitali, Daniele Rosso, Enzo Montoneri, Walter Chitarra, Silvia Tabasso, Marco Ginepro and Claudio Lovisolo. 2015. Sustainable maize production by urban biowaste products. International Journal of Agronomy and Agricultural Research. 6(4): 75-91.

BIS. 1993. Indian Standard Specification IS: 3025. Methods of Sampling and Test (Physical and Chemical) for Water and Wastewater. Part 44: Biochemical Oxygen Demand (BOD).2009. Bureau of Indian Standards, New Delhi (ND).

Bray, R.H. and Kurtz, L.T. 1945. Determination of total, organic and available forms of phosphorus in soils. Soil Science. 59(1): 39-46.

Creed, J.T., Brockhoff, C.A. and Martin, T.D. 1994. Method 200.8 Determination of trace elements in waters and wastes by inductively coupled plasma-mass spectrometry. US Environmental Protection Agency, Cincinnati $(\mathrm{OH})$.

Enujeke, E.C. 2013. Effect of poultry Manure on Growth and Yield of improved Maize in Asaba Area of Delta State, Nigeria. IOSR Journal of Agriculture and Veterinary Science. 4(5): 24-30.

Farhad, W., Saleem, M.F., Cheema, M.A. and Hammad, H.M. 2009. Effect of poultry manure levels on the productivity of spring maize (Zea mays L.). The Journal of Animal \& Plant Sciences. 19(3): 122-125.

Islam, M.A., Islam, S., Akter, A., Rahman, M.H. and Nandwani, D. 2017. Effect of Organic and Inorganic Fertilizers on Soil Properties and the Growth, Yield and Quality of Tomato in Mymensingh, Bangladesh. Agriculture. 7(18): 1-7.

Jackson, J.B. and Dutton, P.L. 1973. The kinetic and redox potentiometric resolution of the carotenoid shifts in Rhodopseudomonas spheroides chromatophores: Their relationship to electric field alterations in electron transport and energy coupling. Biochimica et Biophysica Acta (BBA) - Bioenergetics. 325 (1): 102-113.

Jayasinghe, P.S., Pahalawattaarachchi, V. and Ranaweera, K.K.D.S. 2016. Effect of Seaweed Liquid Fertilizer on Plant Growth of Capsicum annum. Discovery. 52(244): 723-734.

Ji, R., Dong, G., Shi, W. and Min, J. 2017. Effects of Liquid Organic Fertilizers on Plant Growth and Rhizosphere Soil Characteristics of Chrysanthemum. Sustainability. 9(5): 841- 857.

Kalbani, F.O.S.A., Salem, M.A., Cheruth, A.J., Kurup, S.S. and Senthilkumar, A. 2016. Effect of some organic fertilizers on growth, yield and quality of tomato (Solanum lycopersicum). International Letters of Natural Sciences. 53: 1-9.

Kirk, P.L. 1950. Kjeldahl method for total nitrogen. Anal. Chem. 22 (2): 354358.

Martin, T.D., Creed, J.T. and Brockhoff, C.A. 1994. Method 200.2 Sample preparation procedure for spectrochemical determination of total recoverable elements. US Environmental Protection Agency, 
Cincinnati $(\mathrm{OH})$.

Melissa Herman. 2011. Inorganic Fertilizer vs. Cattle Manure as Nitrogen Sources for Maize (Zea mays L.) in Kakamega, Kenya. The Journal of Undergraduate Research at Ohio State. 2: 14-22.

Okoroafor, I.B., Okelola, E.O., Edeh, O., Nemehute, V.C., Onu, C.N., Nwaneri, T.C. and Chinaka, G.I. 2013. Effect of Organic Manure on the Growth and Yield Performance of Maize in Ishiagu, Ebonyi State, Nigeria. IOSR Journal of Agriculture and Veterinary Science. 5(4): 28-31.

Olsen, S.R., Cole, C.V., Watanabe, F.S. and Dean, L.A. 1954. Estimation of Available Phosphorus in Soils by Extraction with Sodium Bicarbonate. U.S. Government Printing Office, Washington (WA) (Circular of the United States Department of Agriculture, 939).

Oyedeji, S., Animasaun, D.A., Bello, A.A. and Agboola, O.O. 2014. Effect of NPK and Poultry Manure on Growth, Yield, and Proximate Composition of Three Amaranths. Journal of Botany.
1-6.

Rice, E.W., Baird, R.B., Eaton, A.D. and Clesceri, L.S. 2012. Standard Methods for the Examination of Water and Wastewater. Amer Public Health Assn, Washington (WA).

Roy, M., Karmakar, S., Debsarcar, A., Sen, P.K. and Mukherjee, J. 2013. Application of rural slaughterhouse waste as an organic fertilizer for pot cultivation of solanaceous vegetables in India. International Journal of Recycling of Organic Waste in Agriculture. 2(6): 1-11.

Stanford, S. and English, L. 1949. A use of flame photometer in rapid soil tests of potassium and calcium. Agron. J. 41: 446-447.

Subbiah, B.V. and Asija, G.L. 1956. A rapid procedure for the determination of available nitrogen in soils. Current Science. 25: 259-260.

Walkey, J.A. and Black, J.A. 1934. Estimation of organic carbon by the chromic acid titration method. Soil Sci. 37: 29-31.

\section{How to cite this article:}

Meignanalakshmi, S., M. Charulatha, C. Vennila, P. Tensingh Gnanaraj, A. Serma Saravana Pandian and Vijayarani, K. 2018. Evaluation of Bovine Digesta as Organic Fertilizer in Maize Using Pot Experiments. Int.J.Curr.Microbiol.App.Sci. 7(07): 506-516. doi: https://doi.org/10.20546/ijcmas.2018.707.062 